\title{
Soft-decision error control for h.f. data transmission
}

\author{
P.G. Farrell, B.Sc., Ph.D., C.Eng., M.I.E.E., and R.M.F. Goodman, B.Sc., Ph.D., C.Eng., M.I.E.E.
}

Indexing terms: Errors and error analysis, codes and decoding, Algorithms

\begin{abstract}
This paper is concerned with the soft-decision decoding of error-correcting codes in the context of h.f. data transmission. The use of soft-decision information from the data modem results in an improvement in the performance of a forward-error-correction scheme, when compared with hard-decision decoding, without any further redundancy penalty. In the paper, we estimate the theoretical improvements that can be expected from soft-decision decoding of block and convolutional codes, in terms of both random and burst error-correcting power. Also, this is related to expected coding gains for the Gaussian and Rayleigh fading channels. In addition, we investigate the performance of soft-decision decoding on two types of h.f. modem: a multisubcarrier parallel transmission format (p.t.f.) type modem, and an experimental serial transmission s.m.i.d.d. type modem. We conclude that significant improvements in the performance of coded systems are obtainable through the use of soft-decision decoding.
\end{abstract}

1

Introduction

In a hard-decision error-control-coded binary data transmission system the receiver/demodulator makes a hard 0/1 decision on each incoming data signal before feeding the demodulated bit to the error-correction decoder. For example, in a multiphase modulation system a 'hard' decision is made at each phase boundary. This procedure results in a degradation of the channel decoder's performance. A soft-decision demodulator, on the other hand, assigns a 'confidence' value to each output bit, in addition to the 'hard' binary 0 or 1 decision. In essence this means that each demodulated bit is quantised into $Q>2$ levels, rather than $Q=2$ levels as in the hard-decision case. This confidence information can then be used to improve the error-correction decoder's performance (in terms of lower output bit error rate) without incurring any further redundancy penalty.

The use of soft-decision decoding is therefore particularly pertinent to the case of h.f. data transmission. This is because, due to the high channel error rates and the nonGaussian error statistics that exist on most h.f. data links, hard-decision decoding schemes simply do not have sufficient error-correction power per bit to provide useful coding gain. Soft-decision decoding schemes, however, can increase the correction power per bit, but at the expense of further complexity. The increase is coding gain (over that achievable with hard-decision) that can be expected by using soft-decision decoding depends on several factors, including the number and spacing of the quantisation levels, the decoding algorithms used, and the channel characteristics.

The performance advantage of using soft-decision decoding for digital data transmission is clear and well quantified for channels perturbed by additive Gaussian white noise (e.g. the space channel). It is not so clear what the advantage of soft-decision decoding is when the channel is subject to fading, multipath, and other multiplicative noise effects (e.g. the h.f. channel). This paper is a contribution towards a qualitative evaluation of the performance advantage of soft-decision techniques when applied to fading h.f. channels. The results seem to indicate that soft-decision enhances the performance of a modulation and coding

Paper $941 \mathrm{~F}$, first received 6 th March and in revised form 2nd July 1980

Dr. Farrell is with the Electronics Laboratories, The University of Kent, Canterbury CT2 7NT, England, and Dr. Goodman is with the Department of Electronic Engineering, The University, Hull HU6 7 RX, England system considerably more when the channel is subject to fading than when it is subject to white noise, in terms of signal/noise ratio (s.n.r.) advantage. It will be shown later, in fact, that a soft-decision decoder can (at most) double the correction power of a code, and therefore achieves a performance that tends to that of the optimum maximumlikelihood decoder. This increase in power is certainly worth having, particularly in the case of h.f. data transmission.

The main objection to the use of soft-decision decoding is one of hardware complexity. This is because in addition to the decoder having to handle and store $J$ bits (where $2_{-}^{J}$ $=Q$ ) instead of 1 bit per decision, existing soft-decision algorithms are much more complex than hard-decision algorithms. This is particularly true in the case of block codes, as opposed to convolutional codes where the Viterbi algorithm provides an effective (although still severely complexity limited) soft-decision decoding scheme. In this paper we investigate the performance of several block and convolutional soft-decision decoding schemes, with the accent on low decoder complexity.

This paper, which is a conflation and extension of our previous work ${ }^{28,29}$ develops in the following way. Firstly, soft-decision decoding is described. Secondly, we estimate the performance improvements that can be expected from soft-decision decoding of block and convolutional codes in terms of both random and burst error-correcting power. Also, this is related to expected coding gains for the Guassian and Rayleigh fading channels. The characteristics of the h.f. channel which have major influences on the choice of any soft-decision error-control scheme are then discussed. Finally, the performances of the schemes used on h.f. channels are assessed, by means of decoding simulations with recorded error data, and via real time h.f. trials.

\section{Soft-decision decoding}

Instead of making a hard decision, on each binary signal received, a soft-decision detector first of all decides whether it is above or below the decision threshold, and then computes a 'confidence' number which specifies how far from the decision threshold the demodulator output is. This number could in theory be an analogue quantity, but in practice, if it is to be useful it must be quantised. Thus the output of the detector is still quantised, but into many more than the two regions of a hard-decision device.

An example of an 8-region device is given in Fig. 1. In this case the input to the demodulator is a binary signal, and the signal space is quantised into eight regions, delin- 
eated by one decision threshold and three pairs of confidence thresholds. Each input signal is thus demodulated to give an output character consisting of one binary hard decision digit and two binary confidence digits. In general, a character consisting of $\log _{2} Q$ binary digits emerges at the output of the demodulator, if there are $Q$ regions in the quantised output signal space (see Fig. 2). Normally $Q$ is a power of 2 . A binary code word of $n$ digits is thus represented by $\log _{2} Q=J$, say, binary code words, each of $n$ digits. One of these consists of the (hard) decision digits, the others consist of the confidence digits of appropriate weighting. If the signal falls in a region of complete confidence, then the confidence digits of the corresponding output character are all ones. The soft-decision distances $d_{s}$ between an output character and each of the two highest confidence output characters are then the Euclidean distances between the output character and each of the highest confidence characters; the distance between characters corresponding to adjacent regions being unity. Thus, for example, if a signal is demodulated as 000 , then the softdecision distances to 111 and 011 are, respectively, 4 and 3 (see Fig. 1). A convenient way of computing the softdecision distance between two characters, when the regions and characters are mapped as in Fig. 1 is to invert the confidence digits of one of the characters if their decision digits are different, then modulo- 2 add the resulting characters, and finally interpret the result as a binary number.

Thus,

$$
d_{s}[000, \quad 111]=000 \oplus 100=100 \equiv 4
$$

and

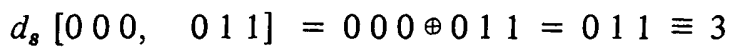

The soft-decision distance (s.d.d.) between a received code word and a possible transmitted code word may be computed by summing the appropriate soft-decision distances for each digit (character) of the code word. Thus, by computing the soft-decision distances to all possible code the one most likely to have been transmitted may be determined by selecting the one with lowest s.d.d. in a manner

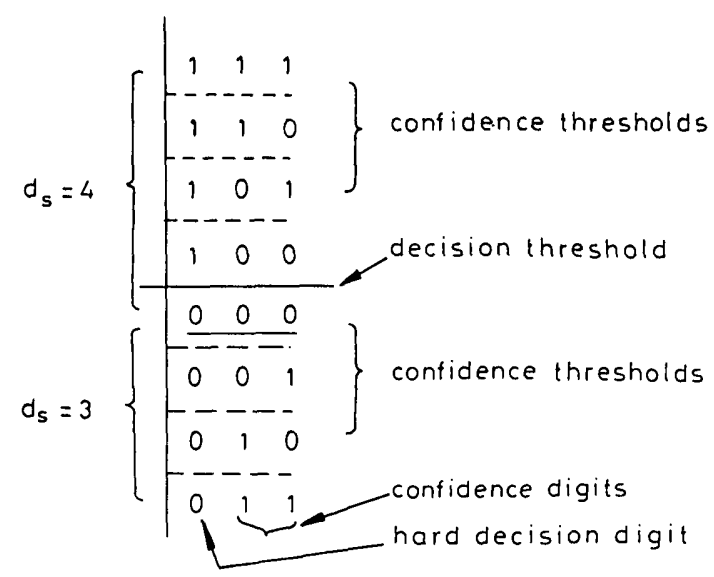

Fig. 1 8-region soft-decision quantisation

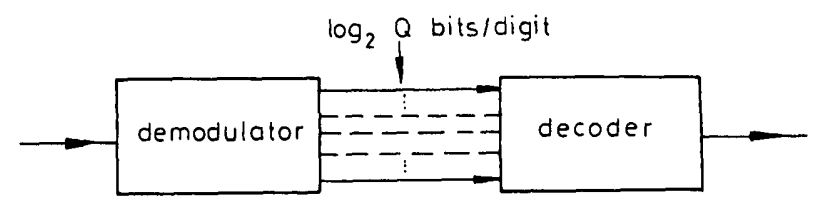

Fig. 2 Soft-decision demodulator and decoder exactly analogous to minimum Hamming distance decoding. ${ }^{1}$ For example, using the demodulator arrangement of Fig. 1 together with a single repetition code, assume that the code word 11 is transmitted. Imagine that the first one is detected as 000 , and the second one as 110 . Then the s.d.d. to $111-111$ is $4+1=5$, and the s.d.d. to $011-011$ is $3+6=9$ (see Fig. 3). The minimum s.d.d. value is 5 , so the soft-decision decoder outputs the correct code word, 11 , in spite of the error in the first digit. A hard-decision decoder, given 01 , could only detect the error, without being able to correct it. The soft-decision method of decoding described above is called minimum soft-decision distance (m.s.d.d.) decoding. It is equally applicable to block and convolutional codes.

The above example shows that a simple single-errordetecting repetition code (block length $n=2$, Hamming distance $d=2$ ) is capable of single-error-correction when used with soft-decision demodulation $\left(d_{s}=14\right)$. This shows that the error control power of a code is almost doubled by the use of soft-decision detection: an $e$-error-detecting code approximates to an $e$-error-correcting code, or a $t$-errorcorrecting code approximates to a $2 t$-error-correcting code. In general,

$$
\begin{aligned}
d_{s} & =d(Q-1) \\
& \simeq d \cdot Q \text { for large } Q
\end{aligned}
$$

In practice values of $\log _{2} Q$ greater than 4 or 5 (16 or 32 regions) are unnecessary, as the increase in performance is only marginal. ${ }^{2}$ In terms of decoder output error rate, the improvement due to soft-decision detection depends on the particular code and channel error statistics, but one to two orders of magnitude or more decrease in output error rate, for white Gaussian noise channels with error rates in the range $10^{-2}-10^{-4}$, is typical. This corresponds to $1.5-2 \mathrm{db}$ improvement in s.n.r. In non-Gaussian noise the improvement may be considerably greater. ${ }^{3}$

Codem $^{4}$ is believed to be the first example of an appli. cation of soft-decision decoding to the h.f. channel. Softdecision techniques have been very successfully used in conjunction with convolutional codes, as applied to a variety of channels, including the h.f. channel. ${ }^{5}$

\section{Expected improvements in performance due to soft-decision decoding}

Given a binary block or convolutional error-control scheme, assume that the soft-decision demodulator quantises each output digit $v_{i}$ to $2^{J}=Q$ levels, symmetrically spaced about the hard $0 / 1$ decision boundary. The estimate of the $i$ th received binary digit is given by the soft-decision $J$-bit byte:

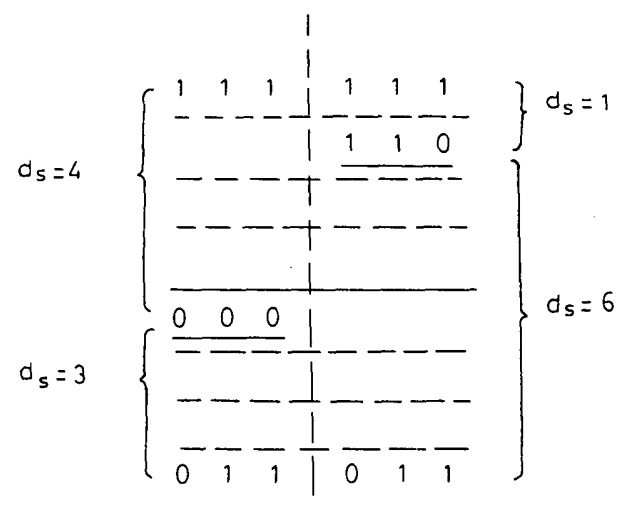

Fig. 3 Soft-decision decoding example 
$\left[v_{i}\right]=\left[v_{i}^{1} v_{i}^{2} \ldots v_{i}^{J}\right]$, where the square brackets indicate a soft-decision quantity. The first bit of $\left[v_{i}\right]$ is the harddegcision estimate, and the remaining $J-1$ bits give an indication of the confidence of that estimate. The confidence of the hard-decision may be defined as the $J-1$ bit byte: $\left[c_{i}\right]=\left[c_{i}^{1} c_{i}^{2} \ldots c_{i}^{J-1}\right]$, where $\left[c_{i}\right]=\left[v_{i}^{2} v_{i}^{3} \ldots v_{i}^{J}\right]$ if $v_{i}^{1}=$ 1or $\left[c_{i}\right]=\left[v_{i}^{2} v_{i}^{3} \ldots v_{i}^{J}\right] \oplus[11 \ldots 1]$ if $v_{i}^{1}=0$. Thus the confidence of a particular received bit can vary from $\left[c_{i}\right]=\left[\begin{array}{llll}0 & 0 & \ldots & 0\end{array}\right]$ (least confident, nearest to the harddecision $0 / 1$ boundary) to $\left[c_{i}\right]=[11 \ldots 1]$ (furthest away from the boundary). Alternatively, we may consider that the demodulator output soft-decision digit $\left[v_{i}\right]$ gives an estimate of the soft-decision error digit $\left[e_{i}\right]$ which has been added to the transmitted digit $\left[u_{i}\right]$. Hence, $\left[v_{i}\right]=\left[u_{i}\right] \oplus$ $\left[e_{i}\right]$, where $\left[u_{i}\right]=[00 \ldots 0]$ or $[11 \ldots 1]$ only. The value of the soft-decision error digit in levels can therefore lie between 0 and $(Q-1)$, and a value of $\geqslant(Q / 2)$ constitutes an 'error' in the hard-decision sense.

An estimate of the improvement in random-error correcting power when soft-decision decoding is used may now be formed. Consider a block or convolutional code whose decoding constraint length is $n$ bits. If the hard minimum distance of the code is $d_{h}$ over $n$ bits, then its boundeddistance hard correcting power is the largest integer $t_{h} \leqslant\{($ $\left.\left.d_{h}-1\right) / 2\right\}$. This gives a per bit hard correction power of $t_{h} / n$. In the soft-decision sense code words (paths) are $\geqslant d_{s}=(Q-1) d_{h}$ soft-decision levels apart, and therefore the bounded distance guaranteed soft-decision errorcorrection power in levels is $t_{s} \leqslant\left\{\left(d_{s}-1\right) / 2\right\}$. The smallest number of levels that constitutes an error in the hard decision sense is $Q / 2$, and the maximum number of 'hard' errors that can be corrected is therefore $t_{s} /(Q / 2)=2 / Q$ $\left\{\left(d_{s}-1\right) / 2\right\}=1 / Q\left\{(Q-1) d_{h}-1\right\} \simeq d_{h}$ for $Q$ large. Thus, the per bit correction power has approximately doubled from $t_{h} / n$ to $d_{h} / n$.

It should be noted that the doubling in correction power is an upper bound on the improvement due to soft-decision, and will only be achieved at very high s.n.r.s. In general, at low s.n.r.s. the average improvement will be significantly less than this.

\subsection{Improvement on the Gaussian channel}

Consider the Gaussian channel. If the (single-sided) noise power density is given by $N_{\mathrm{o}}$, the s.n.r. is given by $\gamma=$ $E / N_{\mathrm{o}}$, and the bit probability of error is given by the $Q$ function

$$
P=\int_{\sqrt{2 \gamma_{b} R}}^{\infty}\left|\exp \left(-x^{2} / 2\right) \sqrt{2 \pi}\right| d x \triangleq Q\left(\sqrt{2 \gamma_{b} R}\right)
$$

where $\gamma_{b}=E_{b} / N_{\mathrm{o}}=\gamma / R$ is the normalised s.n.r. per information bit, and $R$ is the inverse of the bandwidth expansion factor i.e. the code rate). The probability of bit error for a hard-decision coded system can be lower bounded by

$$
P_{e}>w_{d_{h}} p^{d_{h}}(1-p)^{n-d_{h}}
$$

where $d_{h}$ is the minimum distance of the code over the decoding block or constraint length $n$, and $w_{d_{h}}$ is the number of bit errors contributed by incorrect decoding of a code word or path of distance $d_{h}$.

Asymptotically, at high s.n.r., ${ }^{7}$

$$
Q\left(\sqrt{\left.2 \gamma_{b} R\right)} \simeq \exp \left(-\gamma_{b} R\right)\right.
$$

and hence eqn. 2 reduces to

$$
P_{e} \simeq w_{d_{h}} \exp \left(-\gamma_{b} R d_{h}\right)
$$

Assuming that soft-decision decoding effectively doubles the distance of a code, i.e. $d_{s}=2 d_{h}$, then for

$$
P_{e}(\text { soft })=P_{e}(\text { hard })
$$

we require

$$
\gamma_{b}(\text { soft })^{R 2 d_{h}}=\gamma_{b}(\text { hard })^{R d_{h}}
$$

i.e.

$$
\frac{\gamma_{b}(\text { hard })}{\gamma_{b}(\text { soft })}=2
$$

which indicates a $3 \mathrm{~dB}$ improvement in coding gain. This is similar to the improvement obtained in changing from a $d_{h}$ th-order diversity system to a $2 d_{h}$ th-order diversity system. ${ }^{6}$

Also, $P_{e}$ uncoded $\simeq \exp \left(-\gamma_{b}\right)$ from eqn. 3 which shows that the upper limit on coding gain is given by:

$$
\begin{aligned}
G_{c} & <10 \log R d_{h}(\mathrm{~dB}) \text { for hard-decision decoding } \\
G_{c} & <10 \log 2 R d_{h}(\mathrm{~dB}) \text { for soft-decision decoding }
\end{aligned}
$$

At the opposite extreme, i.e. for the very noisy channel, it has been shown that a performance loss of about $2 \mathrm{~dB}$ is incurred when hard-decision decoding is used as opposed to infinitely quantised soft-decision decoding. Also, the degradation involved ${ }^{8}$ in using the much more practical equal-spacing 8-level quantisation is only about $0.2 \mathrm{~dB}$.

Therefore, at high error rates on a Gaussian channel, a maximum soft-decision coding gain of about $1.8 \mathrm{~dB}$ is expected. At low error-rates a $2 \mathrm{~dB}$ improvement in s.n.r. corresponds to a reduction in output bit error rate of approximately two orders of magnitude for uncoded binary antipodal signalling on the Gaussian channel. At high error rates, however, the uncoded performance curve flattens out, and a characteristic of coded transmission is that at some value of $E_{b} / N_{\mathrm{o}}$ a coded transmission will perform worse than an uncoded one. The coded performance curve effectively 'crosses over' the uncoded curve. In this high error rate region, the uncoded output bit error rate is only improved by about a factor of 3 for a $2 \mathrm{~dB}$ improvement in $E_{b} / N_{\mathrm{o}}$. Thus, although we expect an improvement in performance due to soft-decision decoding at high error rates, this improvement will not be large.

\subsection{Rayleigh channel}

The bit error probability for the coherent Rayleigh fading channe ${ }^{6}$ is given by:

$$
P=\frac{1}{2}(1-\mu)
$$

where

$$
\mu=\frac{\gamma_{b} \cdot R}{\gamma_{b} \cdot R+1}
$$

The lower bound on bit error probability (eqn. 2) is then given by:

$$
P_{e}>w_{d_{h}}\left(\frac{1-\mu}{2}\right)^{d_{h}}\left(\frac{1+\mu}{2}\right)^{n-d_{n}}
$$


which for high s.n.r.s becomes:

$$
P_{e} \simeq w_{d_{h}}\left(\frac{1}{4 \gamma_{b} R}\right)^{d_{h}} \simeq K\left(\frac{1}{\gamma_{b}}\right)^{d_{h}}
$$

Assuming $d_{s}=2 d_{h}$, then for equal output bit error rate we have

$$
\gamma_{b(\text { hand })}=\gamma_{b \text { (soft) }}^{2}
$$

which shows that the soft coding gain is an increasing function of $E_{b} / N_{o}$, and that soft-decision decoding requires approximately half the s.n.r. (in $\mathrm{dB}$ ) to achieve the same output bit error rate as hard-decision decoding.

Soft-decision decoding on the Rayleigh fading channel is therefore theoretically capable of providing much larger soft-coding gains than in the case of the Gaussian channel. It must be noted again, however, that the expected halving in power requirement will not be achieved at low s.n.r.s.

\subsection{Burst channe/s}

It is not possible to derive a theoretical soft-decision improvement figure for a complicated time-varying channel such as the h.f. channel. In general, the h.f. channel can be considered $^{9}$ to be a diffuse-burst channel in which error bursts of medium to high density are seperated by relatively short gaps with a low density of errors. As such, any coding scheme that is used on the h.f. channel must have both burst- and random error-correction capability. We have already shown that the random error-correction power of a code is improved by the use of soft-decision decoding. It is therefore appropriate to assess the improvement in burst correcting power.

Consider a random error-correcting code with a correction power of $t_{h}$ over a decoding constraint length of $n$ bits. This implies that all bursts of length $b \leqslant t_{h}$ can be corrected. Assuming that, asymptotically, soft-decision decoding doubles the power of the code, then the code will now be able to correct any combination of two or less bursts of length $b \leqslant t_{h}$, or a single burst of length $2 t_{h}$.

Interleaving is a powerful technique which can be used to provide both burst-and-random correction power, whilst still using a random error-correction decoder. If the above basic code is interleaved to a depth of $\lambda$ then the harddecision power of the interleaved code over $n \lambda$ bits is such that any combination of $t_{h}$ or fewer bursts of length $\lambda$ or less, can be corrected. Thus the application of soft-decision should allow (at a maximum) any combination of $2 t_{h}$ or fewer bursts of length $\lambda$ or less to be corrected. It is therefore the multiple-burst correcting power within a given constraint length that is significantly increases by softdecision decoding rather than the single burst correcting power.

A well known ${ }^{10}$ bound on burst correcting capability $b$ relative to error-free guard space $g$, which holds for both block and convolutional codes, is given by

$$
\frac{g}{b} \geqslant \frac{1+R}{1-R}=3
$$

for a $\frac{1}{2}$ rate code.

In general, an interleaved random-error-correcting code does not approach this bound closely. For example, the $(23,12)$ perfrect Golay code has $b=3$ and $g=20$ giving $g / b=6.7$. However, if the use of soft-decision increases the burst capability by only one to $b=4$ on average, then $g / b=$ $4 \cdot 75$, a significant improvement.

If in the limit, we assume that soft-decision decoding can double the single burst correcting power of a code, then

$$
(g / b)_{\text {soft }} \geqslant \frac{1}{2}\left(\frac{1+R}{1-R}\right)
$$

At high error rates the improvement due to soft decision will not be mainly in the single-burst correction capability but rather in the multiple-burst correction capability. This implies that soft-decision will show the most improvement on a diffuse burst channel rather than a dense burst/long guard space channel.

\section{HF channel error characteristics}

The error characteristics experienced in h.f. data transmission depend not only on the channel characteristics at a particular time but also on the type of modem used.

\subsection{Parallel transmission format (p.t.f.) modem}

In this Section we consider a p.t.f. or Kineplex type modem operating at 2400 bits per second with a nominal transmission bandwidth of $3 \mathrm{kHz}$. The data is transmitted in 48 bit parallel blocks or frames, using orthogonal multisubcarrier phase shift keying. Soft-decision information on demodulated bit is available from the modem. ${ }^{22}$

There are several characteristic types of error events due to this modem structure:

(a) random errors

(b) errors which occur in the same place in repeated frames due to stationary frequency selective fading on one or more subcarriers within the band, thus causing isolated repetitive bursts

(c) sweeping frequency selective fades which traverse the band causing errors in repeated frames but in different frame positions

(d) flat fades across the band which cause large bursts of errors.

Fig. $4 a$ shows a particular received sequence of bits, an error being indicated by an asterisk. It can be seen that one particular subcarrier within the frame is experiencing a bad stationary selective fade, and contributing errors in almost every successive frame. Fig. $4 b$ shows a bad flat and selective fading situation which is causing frequent bursts of errors. Fig. $4 c$ shows a received sequence of bits in which two selective fades are sweeping across the band, causing errors in successive frames but at different frequencies. In addition, all the Figures show a varying amount of residual errors.

As a consequence of these error characteristics it can be seen that any coding scheme used on a bit-by-bit basis must have both burst and random error correction power, and that if a random error-correcting code is used, it must be interleaved in both time and frequency. In practice this involves reading the encoded data stream into a 48 by $\Delta$ array, where $\Delta$ is the interleaving depth in frames; and reading out the bit stream to the modem in a diagonal manner. The inverse operation of de-interleaving is performed at the receiver, before feeding bits to the error-correction decoder. Given a fixed parallel frame of 48 bits, there exists a tradeoff between the time and frequency separation of adjacent bits in the de-interleaved stream. For example, if $\Delta=48$, then bits are separated in time by 48 frames, but will have been transmitted on the same subcarrier. Thus, stationary selective fades will cause long bursts of errors in the 
de-interleaved bit stream. For this modem, we consider interleaving to a depth of 8 frames, which gives adjacent bits a separation of 8 bits in time, and $\Delta / 8=6$ bits in frequency across the frame.

\subsection{Serial transmission format (s.m.i.d.d.) modem}

In this Section the error characteristics of an experimental h.f. serial binary data transmission modem ${ }^{11-13}$ are described. The modem incorporates frequency domain redundancy, in that the spectrum of the binary digits to be transmitted is spread by sequence inversion keying of a 15-bit $m$-sequence (see Fig. 5). After the sequence-inversionkeying (s.i.k.) stage, the waveform is passed through an 11stage baseband equaliser, ${ }^{14}$ adjusted so as to minimise the symbol distortion and intersymbol interference arising in the modem due to the various filter characteristics. Transmission is by means of double-sideband a.s.k., with coherent product detection (see Fig. 6). Digital correlation is used to strip off the $m$-sequence; the correlator outputs one hard-decision digit, and three confidence digits (i.e. $Q=16$, $J=4)$. The $m$-sequence digit rate is in the range $1-2 \mathrm{kbit} / \mathrm{s}$. The nominal transmission bandwidth of this soft-decision minimum distance decoding (s.m.i.d.d.) modem was $3 \mathrm{kHz}$, as determined by the transmitter filter.

Provision was made to tape-record, during a number of h.f. trials, both the errors in the $m$-sequence digits (or chips) and in the hard-decision digit at the output of the correlator. The results of computer analysis are given in Tables $1-3$ below, and in Fig. 7. Table 1 gives the results of an analysis of the incidence of runs of consecutive errors in hard-decision digits, and Table 2 is the consecutive error runs profile for this analysis. Each file represents almost $3 / 4$ hour or recorded data. The guard space is the number of error-free digits between errors.

Table 3 gives the results of an analysis of the incidence of bursts of errors in the $m$-sequence digits (chips), and Fig. 7 is the corresponding burst error and consecutive error run profiles for file $\mathrm{MC} 3 / 3$, which is typical.
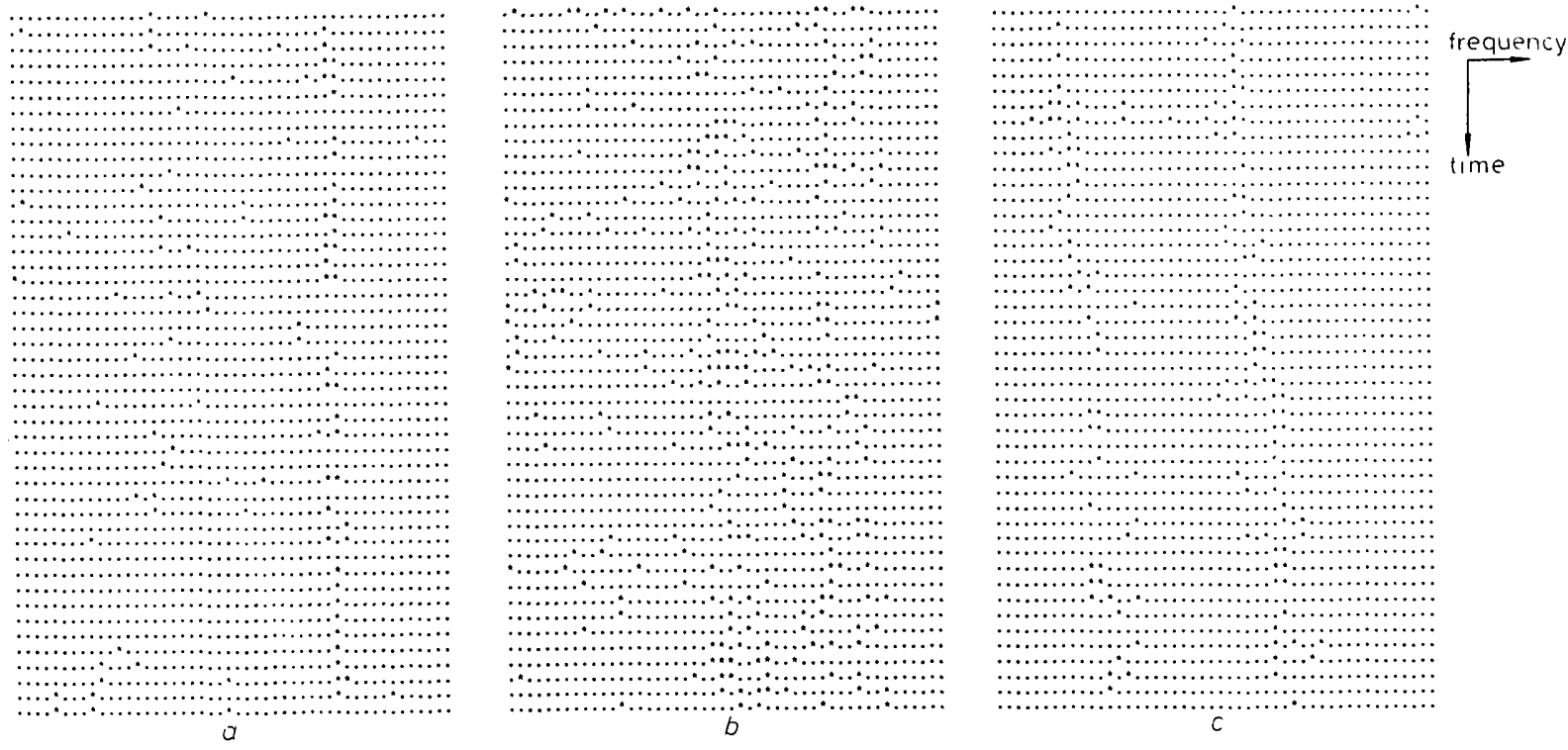

Fig. 4 P.T.F. modem: H.F. error patterns

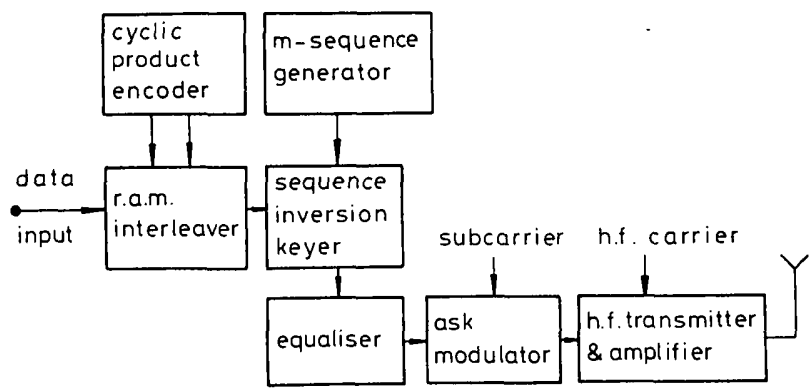

Fig. 5 Experimental s.m.i.d.d. modem: Transmitter

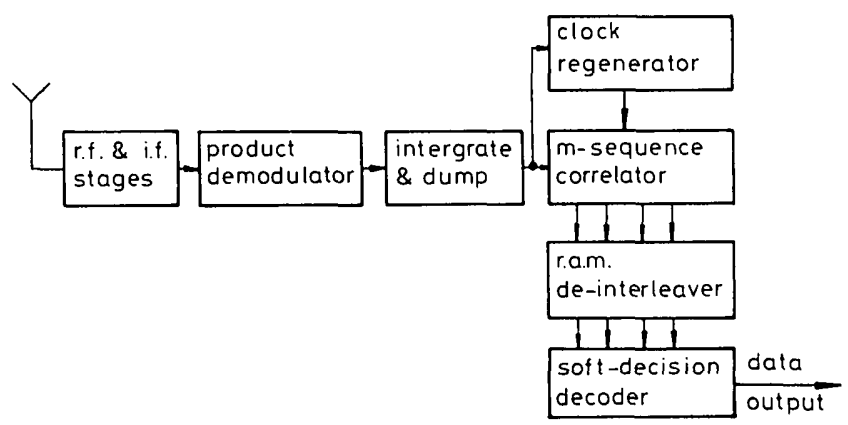

Fig. 6 Experimental s.m.i.d.d. modem: Receiver

Table 1: Consecutive errors in hard-decision digits

\begin{tabular}{llllll}
\hline File number & MC 1/3 & MC 2/3 & MC 3/3 & MC 4/3 & MC 1/6 \\
\hline Number of digits processed & 144000 & 64000 & 1504000 & 47200 & 428800 \\
\hline $\begin{array}{l}\text { Error rate } \\
\begin{array}{l}\text { Average guard space } \\
\text { between runs }\end{array}\end{array}$ & $4.1 \times 10^{-2}$ & $10.85 \times 10^{-2}$ & $2.1 \times 10^{-2}$ & $0.72 \times 10^{-2}$ & $1.36 \times 10^{-2}$ \\
$\begin{array}{l}\text { Average guard space } \\
\text { (discounting single errors) }\end{array}$ & 38.0 & 13.1 & 86.0 & 252.0 & 158.0 \\
$\begin{array}{l}\text { Average number of } \\
\text { consecutive errors }\end{array}$ & 102 & 32.7 & 247 & 810 & 443 \\
\hline
\end{tabular}


Table 2: Errors in hard-decision digits: Consecutive error profile

\begin{tabular}{|c|c|c|c|c|c|}
\hline File number & $\mathrm{MC} 1 / 3$ & $\mathrm{MC} 2 / 3$ & $\mathrm{MC} 3 / 3$ & $\mathrm{MC} \mathrm{4/3}$ & MC 1/6 \\
\hline $\begin{array}{l}\text { Number of runs of } \\
\text { consecutive errors }\end{array}$ & 3636 & 4363 & 1712 & 168 & 2672 \\
\hline \multicolumn{6}{|l|}{$\%$ of runs of length } \\
\hline 1 & $62 \cdot 4$ & 61.9 & 64.9 & $65 \cdot 6$ & $64 \cdot 2$ \\
\hline 2 & $21 \cdot 3$ & $19 \cdot 3$ & $16 \cdot 4$ & $10 \cdot 7$ & 14.4 \\
\hline 3 & 8.94 & 846 & 7.48 & $13 \cdot 10$ & $8 \cdot 30$ \\
\hline 5 & $1 \cdot 79$ & $2 \cdot 25$ & $2 \cdot 51$ & $1 \cdot 78$ & $2 \cdot 66$ \\
\hline 6 & 0.71 & 1.50 & $1 \cdot 28$ & $2 \cdot 38$ & 142 \\
\hline 7 & 0.38 & 0.89 & 0.93 & $1 \cdot 19$ & 1.42 \\
\hline 8 & $0 \cdot 27$ & 0.59 & 1.75 & $1 \cdot 19$ & 1.65 \\
\hline$>8$ & 0.47 & 1.58 & 1.40 & $1 \cdot 19$ & 2.96 \\
\hline
\end{tabular}

Table 3: Bursts of errors in chips ( $m$-sequence digits)

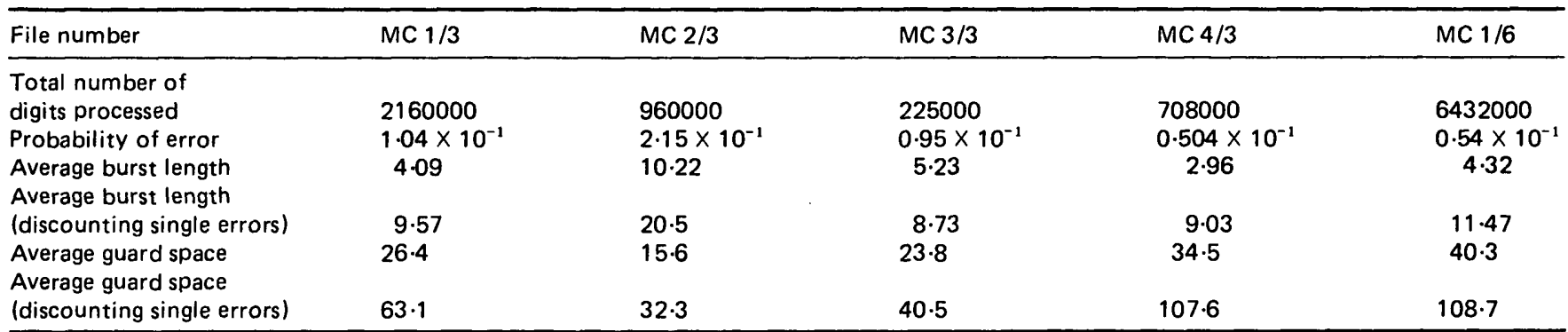

The analysis presented in Table 3 and Fig. 7 is based on a minimum guard space of 4 chips and a burst density threshold of $40 \%$ (i.e. bursts were classified as having an error density of $\geqslant 40 \%$ ).

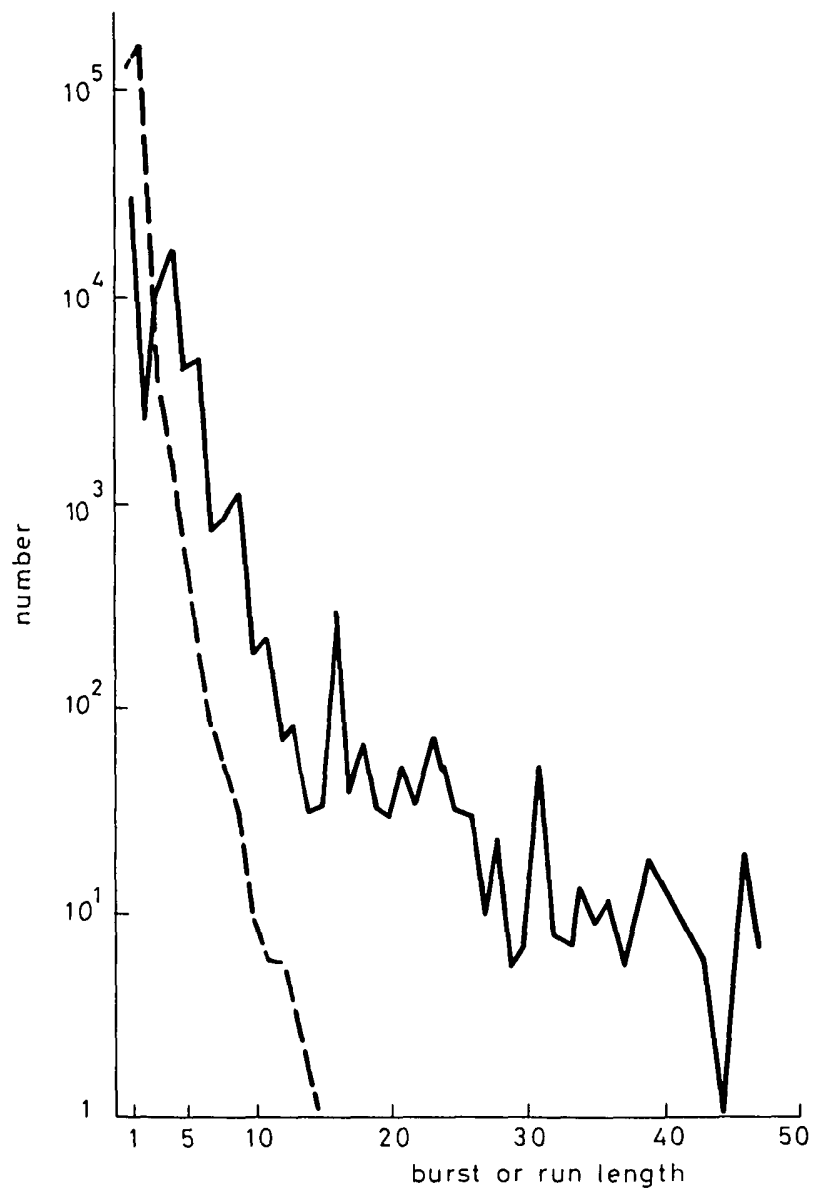

Fig. 7 Chip error profiles on s.m.i.d.d. modem

- - - runs of consecutive errors

bursts of consecutive errors
The diffuse error characteristics of the h.f. channel is apparent in the results of the analysis. Bursts of errors on the chips of the serial s.m.i.d.d. modem are large than those on the parallel subchannels of the p.t.f. modem, as might be expected. The largest burst on the MC $3 / 3$ file is 8658 and there are 494 bursts of length $>50$ chips (see Fig. 7). The effect of $m$-sequence correlation is to make errors in the hard-decision digits more diffuse than the chip errors. This is clear from a comparison of Tables 1 and 2 with Table 4 below; the average run length is less in the chip errors, and the percentage of short $(\leqslant 3)$ runs is higher.

The effect of de-interleaving the hard-decision digits is to effecitvely randomise or diffuse many of the bursts; this, together with soft-decision decoding, enables. the essentially random-error-correcting produce code in the s.m.i.d.d. modem to cope successfully with most of the errors, as will be shown.

\section{Soft-decision decoding schemes}

Given a block or convolutional code operating over a decoding constraint length of $n$ bits, the optimum method of decoding is maximum-likelihood decoding, which, for the binary symmetric channel, is equivalent to minimum distance decoding. A minimum distance hard-decision decoder therefore attempts to find the codeword (path) $u$ nearest in terms of Hamming distance to the received sequence $v$. That is, the code sequence which satisfies

$$
\min \left\{\sum_{i=1}^{n}\left(v_{i} \oplus u_{i}\right)\right\}=\min \left\{\sum_{i=1}^{n} e_{i}\right\}
$$

Alternatively, this is equivalent to finding the minimum weight error pattern $e$ which will turn the received sequence $v$ into a valid code sequence $u$.

Similarly, we may define an optimum soft-decision minimum distance decoder (which approximates to a maximum-likelihood decoder) as one that attempts to find the code sequence at minimum soft-distance (mini- 
Table 4: File MC 3/3: Consecutive error profile

(average run length $=1.26$ chips)

\begin{tabular}{lllllllllll}
\hline $\begin{array}{l}\text { Run length } \\
\text { \% of runs }\end{array}$ & 1 & 2 & 3 & 4 & 5 & 6 & 7 & 8 & 9 & 10 \\
\hline
\end{tabular}

mum number of level errors) from the received sequence $v$. That is,

$$
\min \left\{\sum_{i=1}^{n}\left(\left[v_{i}\right] \oplus\left[u_{i}\right]\right)\right\}=\min \left\{\sum_{i=1}^{n}\left[e_{i}\right]\right\}
$$

We now briefly describe several soft-decision decoding schemes which approximate to this optimum behaviour but also have low complexity. The performance of these codes on the h.f. channel is assessed in the following Section. Each code is identified by an abbreviation.

\subsection{Soft-decision threshold decoding}

Recently, a soft-decision version has been proposed of the well-known hard-decision majority decision threshold decoding algorithm ${ }^{15}$ which is suitable for both block and convolutional codes. ${ }^{16,17}$ The convolutional codes investigated in this class are:

(a) a half-rate random error-correcting code with $n=14$, $t_{h}=2$, and generator sequence $g=11000100000101$ (T14H)

(b) a half-rate random error-correcting code with $n=24$, $t_{h}=3$, and generator sequence $g=1100000000000$ 10100010101 (T24H)

(c) a half-rate burst and random-error-correcting diffuse code $^{18}$ with $n=6 \beta-2$, which can correct any 2 random errors within $n$ consecutive bits, or any bursts of length $\geqslant 2 \beta$ relative to a guard space of $n$ bits. This code has $g=$ $11(00)_{\beta-1} 01(00)_{\beta-1} 01(00)_{\beta} 01$ (TDIFH)

(d) a one-third rate code with $n=15, t_{h}=3$, and $g=$ 111010001001001 (TI5T).

\section{$5.2(23,12)$ perfect Golay code}

The perfect Golay code is triple-error-correcting code with 12 information digits in the deocding constraint length of 23. A soft-decision minimum distance decoding algorithm has been developed for this code, which is based on errortrapping decoding. ${ }^{19}$ The algorithm uses permutation decoding ${ }^{20}$ in a predictive manner such that both burst and random error-correction is possible.

\subsection{Suboptimum soft-decision minimum-distance decoding of convolution codes}

Optimum minimum distance soft-decision decoding of convolutional codes can be achieved by means of the Viterbi algorithm, provided that the encoding constraint length is limited to about 14 bits'so that decoder complexity does not become excessive. Viterbi decoders have been investigated by several researchers, and these have been shown to exhibit reasonably good performance over both satellite and h.f. channels. ${ }^{21,22}$

Recently, however, a hard-decision minimum distance decoding algorithm has been proposed that is efficient for both short and long codes. ${ }^{23}$ The algorithm consists of two main processes: a direct mapping scheme which can locate the minimum distance path without any searching, and an efficient path searching scheme. In this paper we investigate the performance of two very low complexity but suboptimum forms of the algorithm:

(i) hard-decision direct mapping decoding ${ }^{24,25}$

(ii) soft-decision efficient path searching ${ }^{26,27}$

Both decoding methods are applied to the following codes:

(a) a half-rate code with $n=22, t_{h}=3$ and $g=$ $1101000100010001010001(\mathrm{MD} 22 \mathrm{H})$

(b) a one-third rate code with $n=21, t_{h}=4$ and $g=$ 111001010010001011011 (MD2IT)

\subsection{Coding system for s.m.i.d.d. serial modem}

Binary data with rates in the range $33.67 \mathrm{bit} / \mathrm{s}$ is encoded by a half-rate cyclic produce code, and then interleaved (see Fig. 5).

In practice data digits are fed simultaneously into an outer (row) cyclic encoder, into an inner (column) cyclic and into a r.a.m. interleaver, which can store a frame of 900 encoded digits ( 4 complete product code words). This leads to interleaving factors of 56 for the outer code, and 60 for the inner code. Two r.a.m.s are used so that encoding and interleaving can proceed without interruption. The inner and outer codes are $(n, k)=15,11$ codes, with $t_{h}=1\left(d_{h}=\right.$ $3)$, so the complete product code has $(n, k)=(225,121)$ with $t_{h}=4\left(d_{h}=9\right)$.

The character consisting of one hard-decision digit and three confidence digits output by the receiver correlator (see Fig. 6) are fed into a de-interleaving r.a.m. Decoding of the received characters can begin as soon as the r.a.m. is full and synchronisation has been achieved; subsequent detected characters are fed into a second r.a.m., identical with the first, so that reception can continue uninterrupted. Decoding is done by computing the soft-decision (s.d.) distance between each received (possible erroneous) row or column (consisting of 15 4-digit characters) and all the possible correct inner or outer code words. Rows and columns are decoded alternately in order of confidence value. The correct code words are stored in a p.r.o.m. As the distance computations are done sequentially in a sys. tematic order, it is only necessary to store the parity checks of the code words. Also the code is transparent, so that only half the words need be stored away as the remainder are inversions of the first half. When the nearest code word is found, it is read into the r.a.m., via a buffer, replacing the hard-decision digits of the appropriate row or column; all the confidence digits associated with any hard-decision digit which has been inverted during this decoding (i.e. attempted error correction) are reduced to zero. Once all rows and columns have been decoded, then the corrected decision digits can be read out of the r.a.m. There is also provision for a second decoding pass, on rows only. All the processing will take place in the time it takes to fill the second r.a.m., so that the first r.a.m. then becomes free for storing detected characters while those in the second r.a.m. are being processed, and so on. 


\begin{tabular}{lllllll}
\hline & Frames & Bits & Errors & Error rate & $\begin{array}{c}\text { Length, } \\
\mathrm{s}\end{array}$ & Characteristics \\
\hline File 1 & 440 & 21120 & 964 & 1 in 22 & 8.8 & stationary selective fading \\
File 2 & 440 & 21120 & 1951 & 1 in 11 & 8.8 & flat fades \\
File 3 & 440 & 21120 & 1350 & 1 in 16 & 8.8 & sweeping selective fades \\
\hline
\end{tabular}

\section{Performance results}

\subsection{P.T.F. modem}

In this Section, we investigate the performance of the coding schemes described in the preceding Section. The decoding schemes were computer simulated using files of dataindependent soft-decision error sequences, recorded from the modem operating over a real h.f. link. Results are presented for three files which display characteristic error conditions, as summarised in Table 5.

Each coding scheme described in Sections 5.1, 5.2 and 5.3 was tried on each of the three files, using both hard and soft-decision decoding, and both interleaved and noninterleaved operation. Note that the user's data rate depends on the redundancy of the code used, and is equal in this case to either $1200 \mathrm{bits} / \mathrm{s}$ (1/2-rate code) or $800 \mathrm{bits} / \mathrm{s}(1 / 3$ rate code). The results of these simulations are plotted in Fig. 8,9 and 10 , in histogram form, corresponding to files 1,2 and 3 , respectively. The vertical axis corresponds to the normalised relative output bit error for each coding scheme. That is,

$$
\left(\frac{1}{F}\right)=\frac{\text { decoder output data errors }}{\text { channel errors } \times \text { code rate }}
$$

where $F$ can be considered to be the improvement factor in output bit error rate due to the use of coding. Both harddecision and soft-decision results are presented, the unshaded area indicating the soft-decision result and the unshaded plus shaded area indicating the hard-decision result. The shaded area therefore indicates the relative improvement offered by soft-decision over hard-decision decoding. The abbreviations used to identify the coding schemes are as indicated in Section 5. In addition Fig. 11 shows results averaged over all three runs.

From the results presented, it can be seen that softdecision decoding yields useful performance gains for all the coaing schemes tried on the p.t.f. modem error streams. Averaged over all results the output bit error rate is improved by a factor of about $2 \frac{1}{2}$ by the use of softdecision decoding. This result is in excellent agreement with the expected improvement given in Section 3.1, for high error rates.

Comparing the different coding schemes, it can be seen that the more nearly optimum decoding schemes perform best, as expected. For example, with the threshold decoders, the $n=14$ half-rate scheme and the $n=15$ onethird rate scheme both perform reasonable well. This is because threshold decoding performs nearly as well as full minimum distance decoding for short constraint length, low

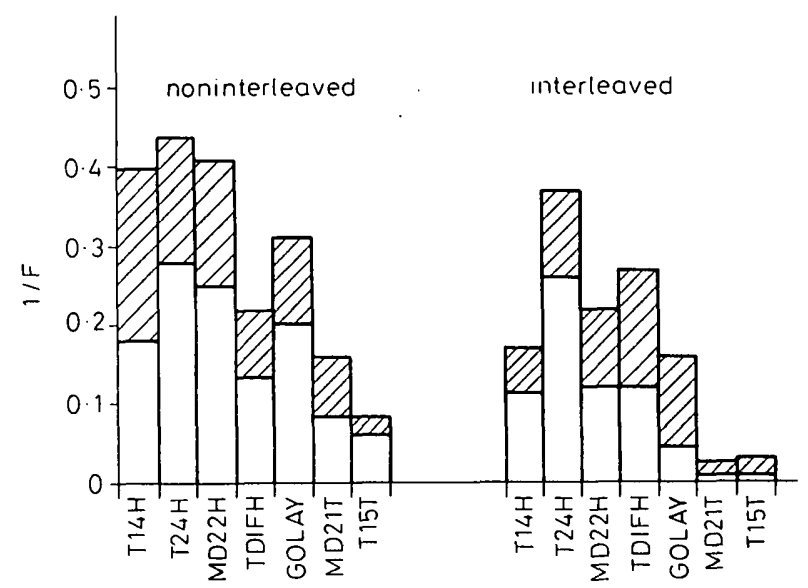

Fig. 8 P.T.F. modem (file 1)

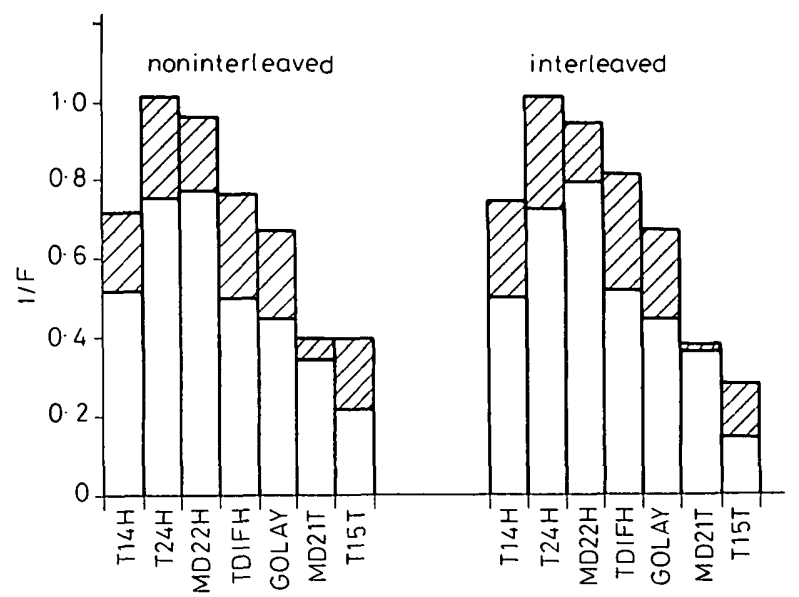

Fig. 9 P.T.F. modem (file 2)

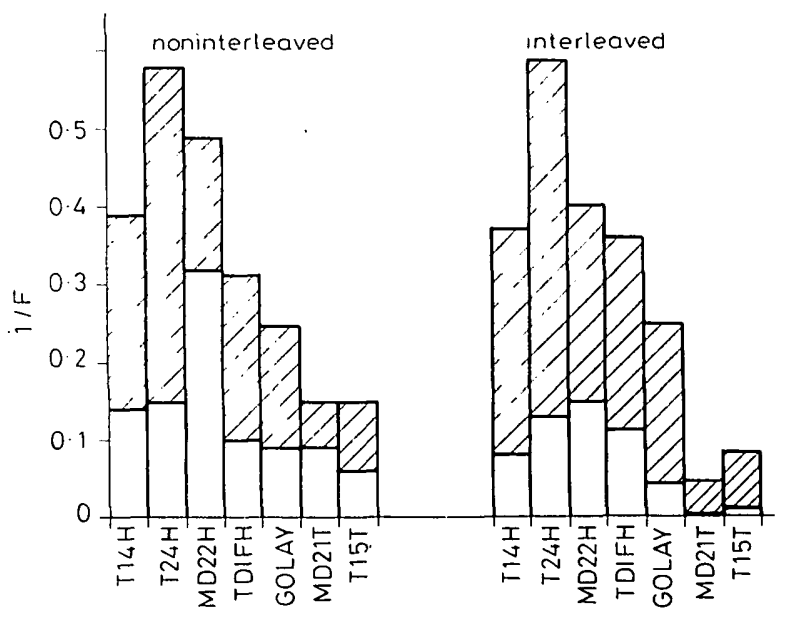

Fig. 10 P.T.F. modem (file 3) 
power codes. As constraint lengths are increased, threshold decoding wastes much of the power of a code, resulting in inferior performance at low error rates. This is exemplified by the $n=24$ half-rate threshold decodeable code. Also, the diffuse decoding scheme, which is essentially low power, performs as well as the interleaved $n=14$. This is to be expected as both schemes have $t_{h}=2$.

In general, as constraint lengths are increased so is correction power. However, this is only true if the decoding scheme utilises the full minimum distance and hence correction power of the code. This can be seen by the way in which the Golay scheme significantly outperforms the suboptimum minimum distance path searching scheme. Both codes operate over roughly the same constraint length, but the Golay scheme is much closer to the optimum maximum

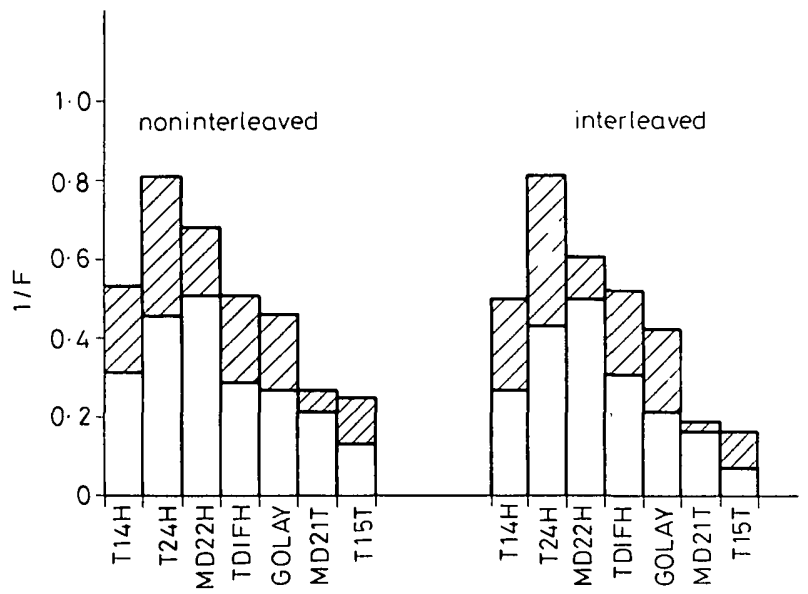

Fig. 11 P.T.F. modem (all files averaged)

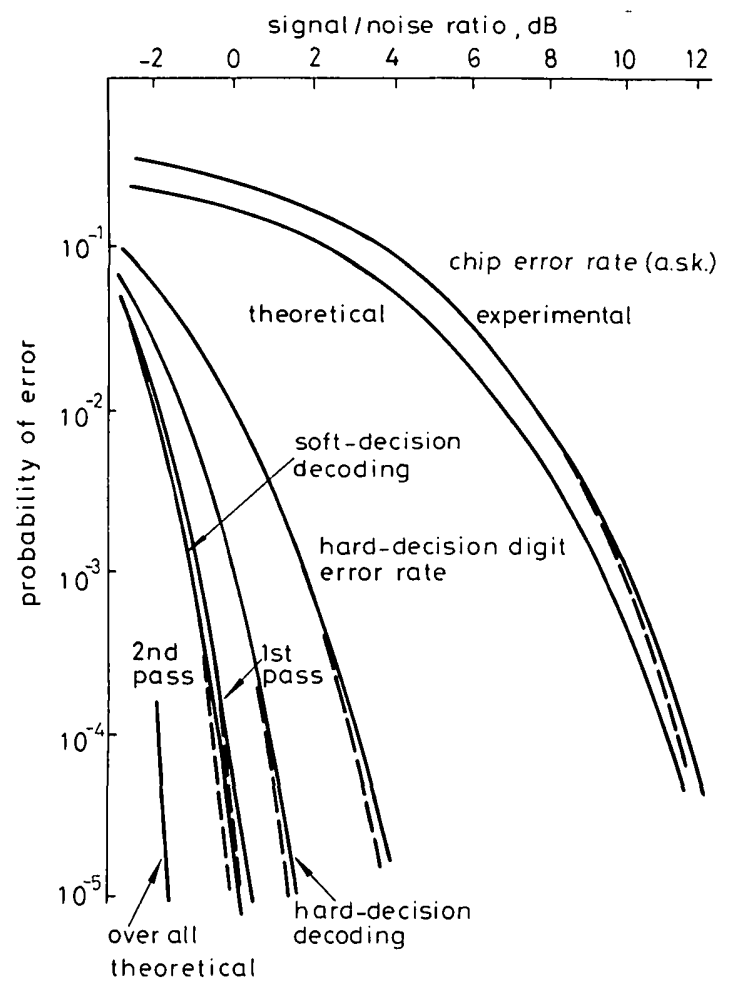

Fig. 12 White noise tests on s.m.i.d.d. modem

$\begin{array}{r}---1.5 \mathrm{Kbit} / \mathrm{s} \\ \hline\end{array}$

IEE PROC., Vol. 127, Pt. F, No. 5, OCTOBER 1980 likelihood decoder over this constraint length. In addition, at high error rates, block codes perform better than convolutional codes, because the output burst of errors due to a decoding is restricted to one block length. A convolutional code may take several constraint lengths to recover from the decoding error, thus causing long output bursts of errors to occur. The minimum distance soft-decision path searching scheme when used on the one-third rate code, however, performs much better than in the half-rate case. This scheme was the only one to achieve zero output errors (file 3).

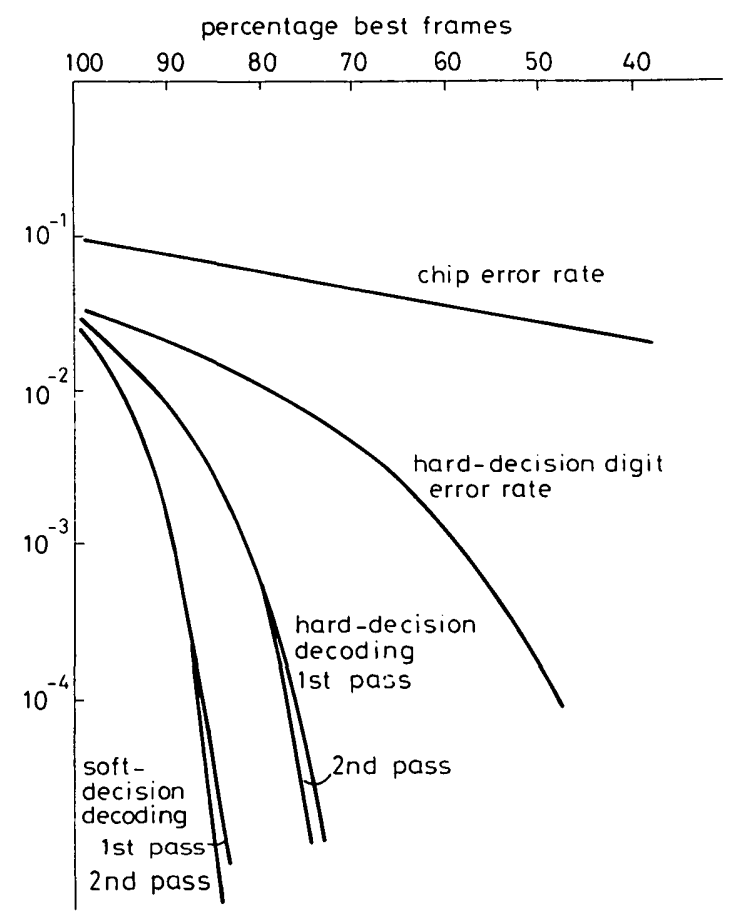

Fig. 13 S.M.I.D.D. modem h.f. trial $160 \mathrm{mile}$ path; $1 \mathrm{kbit} / \mathrm{s}$; all results averaged

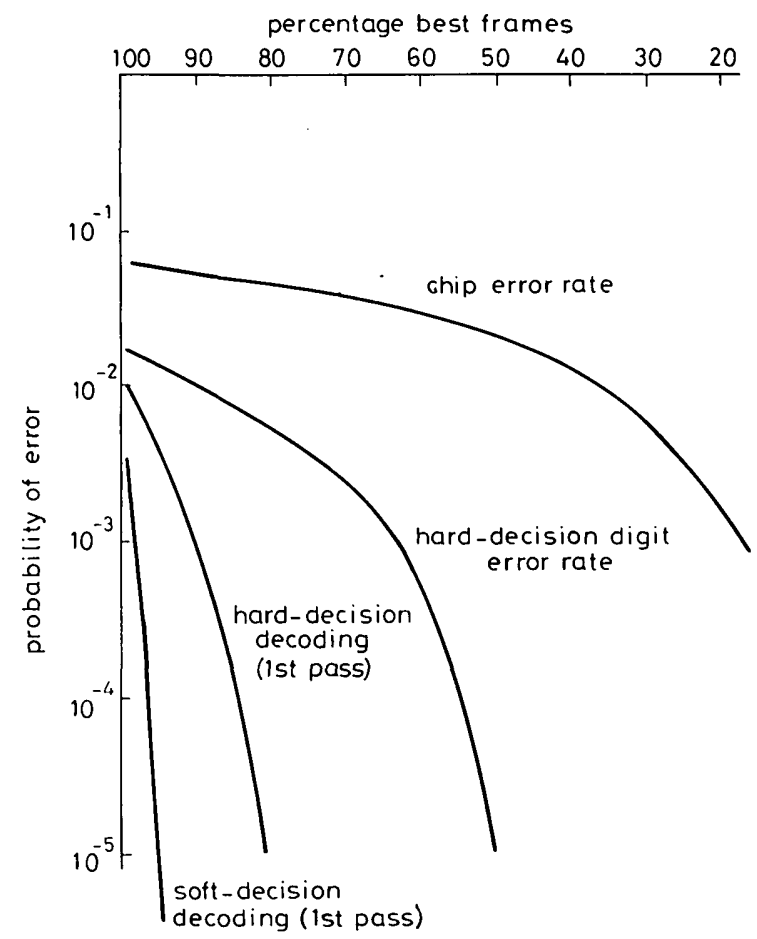

Fig. 14 S.M.I.D.D. modem h.f. trial

$160 \mathrm{mile}$ path, $1 \mathrm{kbit} / \mathrm{s}$, slow fading conditions 


\subsection{S.M.I.D.D. modem}

The performance of the s.m.i.d.d. modem was investigated by means of digital error tests, white noise tests and h.f. field trials. The results of digital random error tests were most satisfactory. For example, a chip error rate of $1.8 \mathrm{x}$ $10^{-1}$ (i.e. the channel error rate) is reduced to $4 \times 10^{-7}$ on the output data digits, after correlation and soft-decision product decoding. ${ }^{13}$ The improvement in error rate over hard-decision decoding is about one order of magnitude in this case. The white noise test (Fig. 12) demonstrates that the system is working within about $1 \mathrm{db}$ of theoretical; that soft-decision decoding gives an increase in performance of about $1.5 \mathrm{db}$ at an output data error rate of $10^{-4}$ and that this in turn is about $2 \mathrm{db}$ better than the performance of the $m$-sequence correlator. Three path lengths $(120,160$ and 300 miles) and three chip rates $(1,1.5$ and $2 \mathrm{Kbit} / \mathrm{s})$ were used for field trials. The average $m$-sequence digit (channel) error rate for all trials is about $10^{-1}$, ranging from about $2 \times 10^{-1}$ for the 300 mile path $(1 \mathrm{Kbit} / \mathrm{s})$ to about $4 \times 10^{-2}$ for the 120 mils path $(1.5 \mathrm{Kbit} / \mathrm{s})$. Output error rates range from about $3 \times 10^{-2}$ to better than $1 \times 10^{-6}$, depending very much on the h.f. channel conditions. Curves of percentage of best frames against output error rate are given in Fig. 13 for all the 160 mile path results averaged, and in Fig. 14 for slow fading conditions on the $160 \mathrm{mile}$ path, both at $1 \mathrm{Kbit} / \mathrm{s}$ chip rate. When the slow fading results are replotted as an s.n.r. against error rate graph (Fig. 15) the separation between the hard-decision and soft-decision product decoding curves is about $6 \mathrm{db}$ at an output error rate of $10^{-4}$. This figure is considerably better than the corresponding figure for white noise tests, thus indicating that the performance advantage of softdecision over hard-decision decoding is much greater under fading channel conditions.

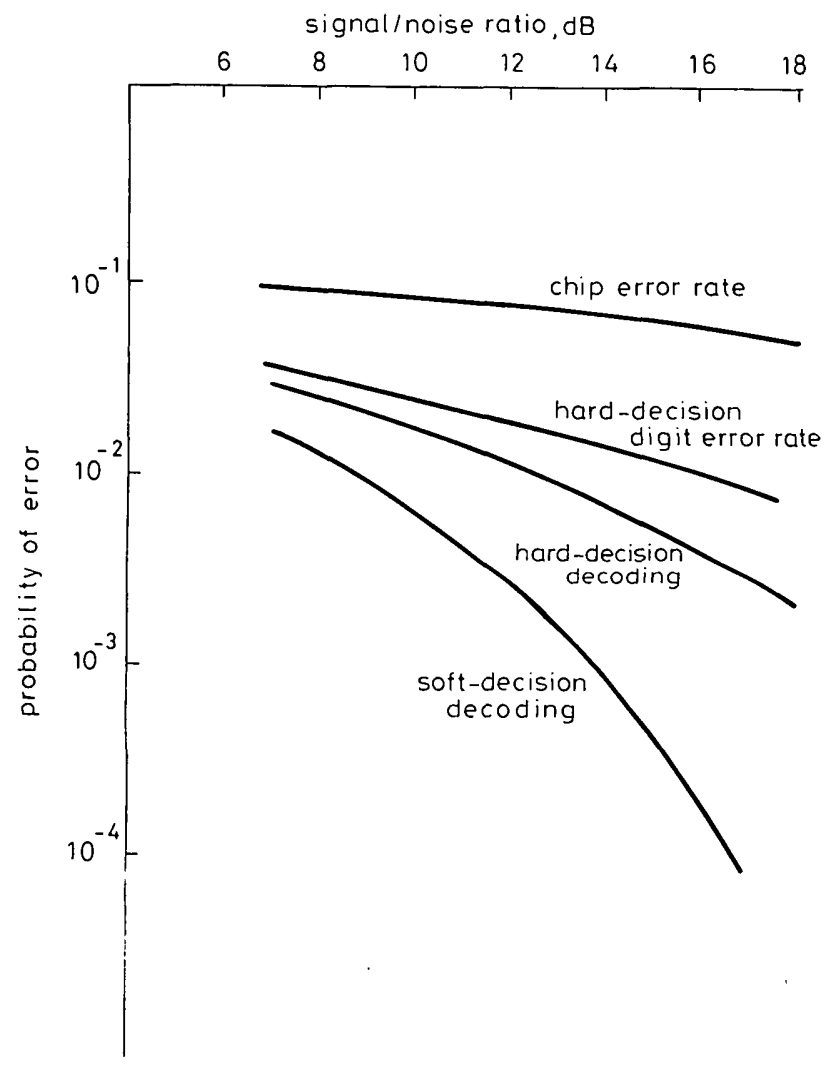

Fig. 15 S.M.I.D.D. modem h.f. trial

$160 \mathrm{mile}$ path, $1 \mathrm{kbit} / \mathrm{s}, 100 \%$ slow fading conditions
Values of $1 / F$ for the half-rate code in the s.m.i.d.d. modem are plotted in Fig. 16, for the h.f. channel conditions of Fig. 14 and 15, and at $100 \%$ and $90 \%$ best frames. These plots confirm the improvement in performance that can be achieved by using soft-decision decoding.

\section{Conclusions}

The main conclusion that can be drawn from this study of the application of error-control coding to h.f. data transmission is that soft-decision decoding gives a valuable increase in performance for only a modest increase in complexity of implementation. The slow fading (Fig. 15) results for the s.m.i.d.d. modem confirm the conclusions in Section 3.2 (on Rayleigh fading), namely, that the softdecision decoding gain over hard-decision decoding is much larger than that achievable in Gaussian noise, that it is an increasing function of s.n.r. and that it is very approxmately half the hard-decision s.n.r. in dB. This last conclusion is only roughly verified because the slow fading $h . f$. channel condition only approximates to Rayleigh fading. Also, the effect of interleaving the the s.m.i.d.d. modem further affects the comparison.

The results in Figs. 8, 9, 10 and 11 for the p.t.f modem confirm the prediction Section 3.3 that soft-decision decoding gives most improvement on a diffuse burst channel rather than on a dense burst/long guard space channel. The flat fading plots (Fig. 9, File 2) almost all indicate worse performance than the corresponding plots for File 1 and File 3. This is also confirmed by the plots in Fig. 16 for the s.m.i.d.d. modem; the $90 \%$ best frames performance is much better than the performance over all frames (100\%), because discounting the $10 \%$ worst frames will remove

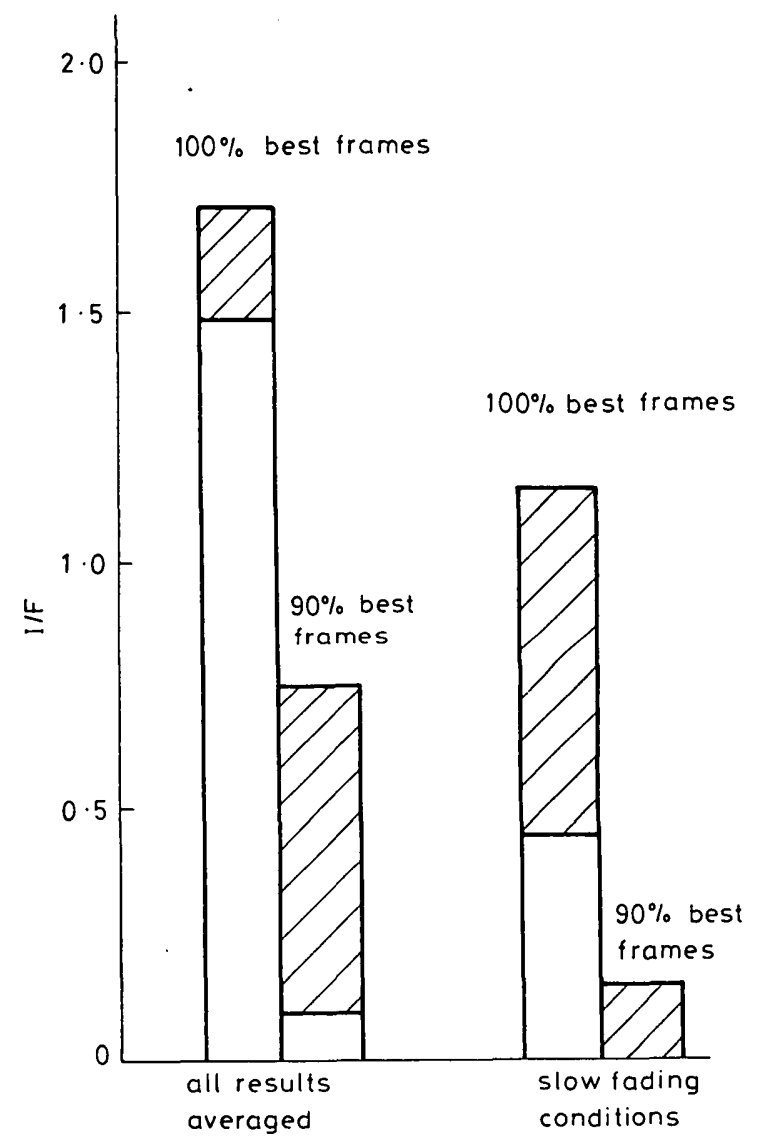

Fig. 16 Performance of s.m.i.d.d. 1/2-rate code $160 \mathrm{mile}$ h.f. path, $1 \mathrm{kbit} / \mathrm{s}$ chip rate 
many dense bursts. It is also interesting to note that interleaving is most effective on a dense burst/long guard space channel, as the plots for stationary selective fading with the p.t.f. modem (Fig. 8, File 1) show; interleaving seems to have relatively little effect on the results for the other files. Again, the s.m.i.d.d. modem results confirm this.

The comparative performance of the various types of code used with the p.t.f. modem indicate that the best results are obtained if an optimum soft-decision decoding scheme is available, for relatively long block or constraint length codes. This requirement implies that powerful, interleaved, random-error-correcting codes should be used, rather than burst-correcting codes which are suboptimum in their random-error-correcting power. Soft-decision Viterbi algorithm decoding of powerful half-rate convolutional codes can give good results, ${ }^{22}$ but optimum soft-decision schemes for powerful half-rate block codes are usually complex to implement. The half-rate code in the s.m.i.d.d. modem has good performance and relatively low complexity, which makes it practical to use, because of the cyclic product structure of the code, together with the efficient adaptive full-minimum-distance soft-decision decoding algorithm. A further implication of the above observation is that soft-decision algorithms should be applied to optimum decoding methods, to minimum distance decoding rather than threshold decoding, for example. Thus the half-rate threshold decoded codes $\mathrm{T} 14 \mathrm{H}$ and $\mathrm{T} 24 \mathrm{H}$ (Section 5, p.t.f. modem) in general do worse than the Golay code, as does the suboptimally decoded convolutional code MD22H (see Figs. 8-11). This disadvantage can be compensated if the code is well matched to the channel error characteristics, as is the half-rate diffuse code TDIFH, which has a performance comparable to that of the Golay code in the noninterleaved case. Interleaving destroys some of its power, as might be expected; its performance in this case is always worse than without interleaving.

The third-rate codes MD21T and T15T have lower decoder output error rates than the half-rate codes, in all cases. It is difficult to evaluate this result, however, because of the different data throughput rates. A true assessment would require different modem/error-correction designs to be compared on the basis of output data bit error rate, with each design operating within a fixed channel bandwidth, and at a fixed data output speed.

It is tempting to try and compare the performance of the serial and parallel modems. This is impossible, however, because of the very different data rates, channel conditions and coding schemes involved. It is interesting, however, to observe that the p.t.f. bit stream before decoding, and the s.m.i.d.d. chip stream before correlation, which have roughly comparable transmission rates, suffer from approximately the same error rate (in the range $10^{-1}$ to $10^{-\hat{2}}$ ). It is hoped that some research in progress at the moment will eventually allow a quantitative comparison of serial and parallel operation, with and without coding, under identical conditions. Further work on soft-decision techniques also includes the development of a full soft-decision minimum distance decoding algorithm based on the algorithm in Reference 23 , which should provide equal or superior results to that of the Viterbi algorithm. In addition, such a decoding scheme would be considerably less complex than a Viterbi decoder, for one-third rate codes.

Finally, it is encouraging to note that efficient softminimum-distance decoding algorithms of relatively low complexity have been found for relatively long $(n \geqslant 100)$ block codes with rates of one half or more.

\section{Acknowledgments}

We would like to thank our colleagues E. Munday (University of Kent at Canterbury), A.D. Green and A.F.T. Winfield (University of Hull), and J. Gordon and N. Montague (Hatfield Polytechnic) for helpful discuissions and valuable assistance.

\section{$9 \quad$ References}

1 PETERSON, W.W., and WELDON, E.J.: 'Error-correcting codes' (MIT Press, 1972)

2 BATSON, B.H.: 'Simulation results for the Viterbi decoding algorithm', NASA report TR R-396, 1972

3 CHASE, D. 'Digital signal design concepts for a time-varying Rician channel', IEEE Trans., 1976, CT-24, pp. 164-172

4 CHASE, D.: 'A combined coding and modulation approach for communication over dispersive channels', ibid., 1973, CT-21, pp. $159-174$

5 MONTAGUE, N.: 'An application of error protection to a vocoded speech channel'. IERE conference on digital processing of signals in communication, Loughborough, Sept., 1977

6 CHASE, D.: 'A ciass of algorithms for decoding block codes with channel measurement information', IEEE Trans., 1972, IT-18, pp. $170-182$

7 WOZENCRAFT, J.M., and JACOBS, I.M.: 'Principles of communication engineering (Wiley, New York, 1965)

8 JACOBS, I.M.: 'Sequential decoding for efficient communication from deep Space', ibid., 1967, COM-15, pp. 492-501

9 BRAYER, K. and CARDINALE, O.: 'Evaluation of error correction block encoding for high speed HF data, ibid., 1967, COM-15, pp. $370-382$

10 GALLAGER, R.G.: 'Information theory and reliable communication' (Wiley, New York, 1968)

11 FARRELL, P.G., and MUNDAY, E.: 'Economical practical realisation of minimum-distance soft-decision decoding for data transmission'. Proceedings of the Zurich international seminar on digital Communication, March 1976

12 FARRELL, P.G., MUNDAY, E., and KALLIGEROS, N.: 'Digital communications using soft-decision detection techniques', AGARD symposium on digital Communication in avionics, Munich, June 1978

13 MUNDAY, E.: 'Soft-decision decoding for HF data communications'. Ph.D. thesis, University of Kent at Canterbury, 1978

14 MUNDAY, E.: 'Phase-shift keying equalisation'. M.Sc. dissertation, University of Kent at Canterbury, 1973

15 MASSEY, J.L.: 'Threshold decoding' (MIT Press, Cambridge, Mass., 1963)

16 GOODMAN, R.M.F., and NG, W.H.: 'Soft-decision threshold decoding of convolutional codes'. IERE Conference Proceedings 37, Sept. 1977

17 GOODMAN, R.M.F.: 'Soft-decision threshold decoders'. Conference proceedings of the international summer school on coding theory, CISM, Udine, Italy, 1978

18 BRAYER, K.: 'Error correction code performance on HF, troposcatter, and satellite channels', IEEE Trans., 1971, COM19 , pp. 781-789

19 GOODMAN, R.M.F., and GREEN, A.D.: 'Microprocessorcontrolled soft-decision decoding of error-correcting block codes'. IERE Conference proceedings 37, Sept. 1977

20 GOODMAN, R.M.F., and GREEN, A.D.: 'Microprocessorcontrolled permutation decoding of block error-correcting codes'. IERE Conference proceedings 41 , Sept. 1978

21 DAVIES, B.H.: 'The implementation of Viterbi decoding on satellite communication circuits'. IERE Conference proceedings 37, Sept. 1977

22 MONTAGUE, N.: 'An application of error protection to a vocoded speech channel'. IERE Conference proceedings 37 , Sept. 1977

23 NG, W.H., and GOODMAN, R.M.F.: 'An efficient minimumdistance decoding algorithm for convolutional error-correcting codes, Proc. IEE, 1978, 125, pp. 97-103

24 WINFIELD, A.F.T.: 'Minimum distance decoding of convolutional error-correcting codes'. Diploma thesis, University of Hull, 1978 
25 GOODMAN, R.M.F.: 'On the design of practical minimum distance convolutional decoders'. Conference proceedings, International summer school on coding theory, CISM, Udine, Italy, 1978

26 NG, W.H.: 'Optimisation of decoding procedures for convolutional codes'. Ph.D. thesis, University of Hull, 1978

27 GOODMAN, R.M.F., and WINFIELD, A.F.T.: 'Soft-decision direct mapping decoding of convolutional codes'. IEEE inter- national symposium on information theory, Grignano, Italy, 1979

28 FARRELL, P.G., and MUNDAY, E.: 'Soft-decision detection of HF signals'. Conference on recent advances in HF communication systems and techniques, IEE, London, Feb. 1979

29 GOODMAN, R.M.F., GREEN, A.D., and WINFIELD, A.F.T.: 'Soft-decision error-correction coding schemes for HF data transmission. Conference on recent advances in HF communication systems and techniques, IEE, London, Feb. 1979 